Rev. Joseph GRZYWACZEWSKI

(Séminaire Polonais de Paris)

\title{
TENDANCES SUBÖRDINACIENNES DANS LA THÉOLOGIE AVANT NICÉE
}

Après la Pentecôte les apôtres se sont mis à la prédication du salut dans le Christ. Ceux qui accueillaient le message apostolique avec foi, recevaient le baptême au nom du Père et du Fils et du Saint-Esprit. La prédication du salut et surtout la tradition de mettre toujours, soit dans la prière, soit dans le baptême, le Père, le Fils et le Saint Esprit sur le même niveau a fait apparaître parmi les chrétiens une question théologique de grande importance: Qui sommes nous? Sommes nous des monothéistes ou peut-être des polythéistes? Est-ce que Dieu est „un" et „unique”? Ou peut être il y a trois dieux différents? Autrement dit: comment joindre la proclamation d'un seul Dieu avec le culte du Fils et du Saint Esprit? Cette question pouvait être facilement posée aux chrétiens par les non chrétiens et surtout par les Juifs. Comme c'était le Christ qui se trouvait en première place dans la prédication, on s'est posé d'abord la question sur Lui, sur sa nature, sa mission, et surtout sur sa relation au Père. La question de l'Esprit Saint allait apparaître plus tard.

Nous avons un écho des questions sur la nature du Christ chez plusieurs auteurs de l'antiquité. St. Justin ( $†$ vers 167) dans son livre Le dialogue avec Tryphon aborde ce problème. Le philosophe chrétien explique au cours du débat:

„Lorsque le Verbe parle du sang de la grappe, c'est un artifice pour montrer que le Christ aura du sang et non point d'une semence humaine, mais de la part de la Puissance de Dieu [...] Cette prophétie montre que le Christ n'est pas un homme d'entre les hommes, engendré suivant le mode ordinaire des hommes"'.

Le Juif Tryphon lui objecte:

„Nous nous souviendrons aussi de cette interprétation que tu donnes là, lorsque tu confirmeras par d'autres preuves encore ton opinion elle-même. Mais présentement, reprends notre sujet et montre-nous que l'esprit prophétique reconnaît qu'il

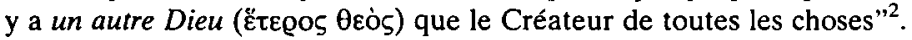

${ }^{1}$ Dialogus cum Judaeo Tryphone 54, PG 6, 593D-596A.

2 Ibidem. 
Voilà, le problème fondamental: quand le chrétien parle de la nature divine du Christ, on pose tout de suite la question: si le Christ a une nature divine, donc il est Dieu; dans ce cas-là il serait „un autre Dieu" par rapport à Dieu le Créateur. Est-ce que c'est possible? Il fallait expliquer comment joindre la divinité du Christ avec le monothéisme. Dans ce contexte on a proposé quelques solutions: l'adoptianisme (le Christ c'est homme qui a été adopté par Dieu), le docétisme (le Christ n'avait pas un vrai corps humain), le modalisme (il n'y a qu'un seul Dieu qui apparaît une fois comme le Père, une autre fois comme le Fils, une fois comme l'Esprit-Saint). C'étaient des réponses simplifiées, on les a rejetées assez tôt.

\section{LE CHRIST COMME LE VERBE DE DIEU}

Frappés par la question: qui est le Christ en lui même et surtout par rapport au Père, poussés par des théories hétérodoxes, les théologiens chrétiens se sont mis à approfondir la vision du Christ comme le Verbe de Dieu, le Logos. Le point de départ de cette théorie était le Prologue de Jean:
„Au commencement était le Verbe et le Verbe était auprès de Dieu et le Verbe était Dieu.
Il était au commencement auprès de Dieu.
Tout fut par lui et sans lui rien ne fut" (Jn 1, 1-2).

Le mot Verbe est la traduction du terme grec Logos. La doctrine du Logos était bien développée dans la philosophie ${ }^{3}$. On a remarqué les points semblables entre le Logos grec et la Sagesse de Dieu de l'Ancien Testament:
„Moi, la Sagesse, j'habite avec le savoir-faire,
je possède la science de la réflexion [...]
A moi appartient le conseil et la prudence,
je suis l'entendement, à moi la puissance [...]
Le Seigneur m'a créée, prémices de son ouvre, avant ses œuvres les plus anciennes.
De l'éternité je fus établie, dès le principe, avant l'origine de la terre [...]
Car qui me trouve, trouve la vie,
il obtient la faveur du Seigneur, mais qui pèche contre moi, blesse son âme, quiconque ma hait chérit la mort" $(\operatorname{Pr} 8,12-16$ et 34-36).

La Sagesse qui se présente dans cette hymne a des traits d'une personne, elle parle d'elle même, elle n'est pas une qualité de Dieu. Elle souligne sa

${ }^{3}$ Cfr. C. Huber, Logos, LThK VI 1119-1128. 
science et son intelligence, et avant tout elle est créée avant toutes les choses, elle fut établie de l'éternité, dès le principe, avant l'origine de la terre, elle était toujours après de Dieu. On a commencé à appliquer cette vision de la Sagesse, ainsi que celle du Logos, au Fils de Dieu. C'est lui qui est la Sagesse de Dieu, c'est lui qui est son Verbe, son Logos, qui s'est fait chair.

L'application de ces deux théories au Christ aidait beaucoup à expliquer sa relation au Père, mais elle produisait une vision descendante, elle plaçait le Fils dans une position inférieure par rapport au Père. Cette façon de voir le Christ est appelée le subordinatianisme. Cela n'était pas une hérésie, mais plutôt une tendance théologique. „Cette tendance est importante dans la théologie du IIe et du IIIe siècle [...]. Le subordinatianisme a surtout été développé par la christologie du Logos. Cette théologie a été influencée par le moyen platonisme; elle considère le Christ comme Logos et Sagesse divine, comme moyen de contact et de médiation entre la divinité transcendante du Père et le monde; aussi pense-t-on que le Christ est dans une position subordonnée par rapport au Père. Quand la réflexion trinitaire inclura le Saint-Esprit (comme chez Origène), celui-ci sera à son tour considéré comme inférieur au Fils"4.

A la base d'une telle façon de voir le Christ, il y a quelques affirmations dans le Nouveau Testament, par exemple: „Le Père est plus grand que moi” (Jn 14, 28); „Quant à ce jour, ou à l'heure, personne ne les connaît, ni les anges dans le ciel, ni le Fils, personne que le Père" (Mc 13, 32). „Abba [Père]! Tout est possible: éloigne de moi cette coupe; pourtant, pas ce que je veux, mais ce que tu veux” (Mc 14, 36). „Un notable l'interrogea en disant: «Bon Maître, que me faut-il faire pour avoir en héritage la vie éternelle»?. Jésus lui dit: «Pourquoi m'appelles-tu bon? Nul n'est bon que Dieu seul» $(\operatorname{Lc} 18,18)$. „Puis ce sera la fin, lorsqu'il remettra la royauté à Dieu le Père, après avoir détruit toute Principauté, Domination et Puissance [...] Mais lorsqu'il dira: «Tout est soumis désormais» c'est évidemment à l'exclusion de Celui qui lui a soumis toutes les choses. Et lorsque toutes les choses lui auront été soumises, alors le Fils luimême se soumettra à Celui qui lui a tout soumis, afin que Dieu soit tout en tous” (1Cor 15, 24-28). „Car Dieu est unique, unique aussi le médiateur entre Dieu et les hommes, le Christ Jésus, homme lui-même, qui s'est livré en rançon pour tous" (1Tm 2, 5).

Ces textes pris à la lettre présentent le Christ comme inférieur par rapport au Père: chez Jean, le Christ constate lui-même que le Père est plus grand que

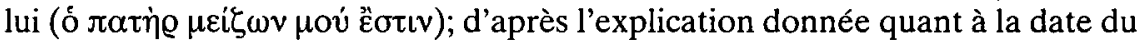
jour du Seigneur, on peut comprendre que même le Fils ne la connaît pas, mais seulement le Père, alors le Père est plus important que le Fils. A Gethsémani le Christ se soumet à la volonté du Père, en soulignant: „pas ce que je veux, mais ce que tu veux", alors il semble que le Père est plus important ou supérieur que

\footnotetext{
${ }^{4}$ M. Simonetti, Subordinazionismo DPAC II 3327.
} 
le Fils. D' après Luc, Jésus semble faire opposition entre lui-même et son Père, en disant que nul n'est bon que Dieu seul (le Père). Dans le même sens on peut comprendre l'explication de St Paul qui déclare que le Christ remettra la royauté à Dieu le Père et se soumettra au Père ${ }^{5}$. Dans la lettre à Timothée, St Paul semble ouvertement déclarer qu'il n'y qu'un seul Dieu, c'est-à-dire le Père et que Jésus Christ est un homme et un médiateur entre Dieu les hommes.

Ainsi, „dans les explications des relations entre le Fils (le Logos) et le Père, nous trouvons chez les théologiens avant le concile de Nicée des tendances subordinatiennes. On les voit chez Justin, Athénagore, Théophile, Tertullien, Hippolyte, Novatien, Origène et chez d'autres. D'après ces théologiens le Logos a existé toujours comme la Raison et la Sagesse dans le Père (logos endiathetos). Pour la création du monde, il est apparu à l'extérieur comme le médiateur entre le Dieu invisible et la créature (logos prophorikos), sans devenir une créature lui-même. Pour les théologiens de l'époque il était difficile d'expliquer la nature du Fils autrement que dans le cadre d'une vision descendante platonicienne et néo-platonicienne, où le Logos se présentait comme un autre Dieu où comme une partie de la Divinité. Aussi des motifs gnostiques, aristotéliques et des éléments du monothéisme judaïque y sont à prendre en considération" 6 . Un autre facteur qui semblait justifier la vision subordinatienne était „la présentation des théophanies de l'Ancien Testament comme logosphanies, ainsi que l'habitude de souligner la grandeur du Père et la temporalité du Fils qui exécute la volonté du Père [...] le subordinatianisme, un événement chrétien, bien que influencé par les courants philosophiques, exprime l'atmosphère de cette époque"7.

\section{VISION DESCENDANTE D'ORIGENE}

1. Dans la doctrine sur le Christ. Bien que plusieurs théologiens étaient marqués par la tendance subordinatienne, elle est surtout visible chez Origène. Il ne faut pas oublier qu'il donnait ses explications sur la relation du Fils au Père dans le contexte des polémiques avec les monarchiens et modalistes. Dans un de ses livres, il dit:

„Dans nos prières, maintenons avec les uns la dualité et en même temps introduisons avec les autres l'unité; et ainsi, d'une part, nous ne tombons dans l'opinion de ceux qui se sont séparés de l'Eglise pour verser dans l'illusion de la monarchie, supprimant le Fils en le retirant au Père et virtuellement supprimant en même temps le Père. D'autre part, nous ne tombons pas dans une autre doctrine impie,

\footnotetext{
${ }^{5}$ D'autres textes à comparer: Ac 2, 36; Phil 2, 5-11; Hbr 1, 4; 3, 11; 5, 1-10.

${ }^{6}$ M. Schmaus, Katholische Dogmatik, I, München 1960, 423, trad. propre.

7 W. Marcus, Subordinatianismus, LThK IX 1138, trad. propre.
} 
celle qui nie la divinité du Christ. Que veulent donc dire les saintes Ecritures, par exemple dans ce passage: "Avant moi il n'y a pas d'autre Dieu, et il n'y en aura pas après moi» (Is 43,10 ), et dans celui-ci: «C'est moi qui suis et il n'y a pas d'autre Dieu que moi» (Dt 32,39). Dans ces formules, il ne faut pas croire que l'unité s'applique au Dieu de l'univers séparé du Christ, et pas davantage au Christ séparé de Dieu. Disons qu'il en est comme dans la parole de Jésus: «Mon Père et moi nous sommes un» (Jn 10, 30)"8.

Origène rejette totalement les opinions des adoptianistes, c'est à dire de ceux qui voulaient voir en Christ un homme choisi par Dieu pour une mission spéciale et à cause de cela appelé fils de Dieu ou même Dieu, mais c'est au sens allégorique ou par adoption. Avec la même vigueur, il s'est opposé aux monarchianistes qui identifiaient le Fils avec le Père, ils privaient le Fils de la divinité et de l'existence réelle, parce qu'ils disaient que c'est le Père lui-même qui s'était manifesté et même c'était lui qui avait souffert; ensuite ils niaient la paternité de Dieu, parce que si Dieu n'a pas vraiment un Fils il n'est pas un vrai Père. Ainsi, selon les monarchianistes - nous montre Origène - le Christ n'est pas vrai Dieu, ni un vrai Fils, et Dieu le Père n'est pas un vrai Père. Les passages de la Bible où il est dit qu'il y a un seul Dieu doivent être compris au sens: Dieu est un, mais uni avec son Fils.

Quand on parle que le Fils est né du Père, on risque de tomber dans une autre erreur: supposer qu'il y avait un temps ou le Fils n'existait pas. Cela signifierait aussi qu'il y avait un temps où Dieu n'était pas Père, mais il l'est devenu quand le Fils était né. Origène rejette une telle hypothèse:

„Comment peut-on penser et croire, si on veut savoir et penser pieusement de Dieu, que Dieu le Père ait jamais été, même un petit moment, sans engendrer cette Sagesse. Ou l'on dira que Dieu n'a pas pu engendrer cette Sagesse avant qu'il l'ait engendrée, de sorte qu'il a mis au monde ensuite ce qui n'existait pas auparavant, ou bien qu'il pouvait, certes, l'engendrer, mais, supposition qu'on ne doit pas faire, qu'il ne le voilait pas. Car l'une est l'autre hypothèse sont absurdes et impies, cela est clair, qu'on imagine qu'il ait progressé de l'impuissance à la puissance, ou que, pouvant le faire, il ait négligé et différé d'engendrer la Sagesse. C'est pourquoi nous savons que Dieu est toujours le Père de son Fils unique, né de lui, tenant de lui ce qu'il est, sans aucun commencement [...]. Il faut donc croire que la Sagesse a été engendrée sans aucun commencement qu'on puisse affirmer ou penser. Dans cet être subsistant de la Sagesse était virtuellement présente et formée toute la création future, que ce soit les êtres qui existent en premier lieu, que ce soit les réalités accidentelles et accessoire, tout cela préformé et disposé en vertu de la prescience"".

Ainsi Origène souligne que le Fils appelé Sagesse existe éternellement, sans commencement. On ne peut pas s'imaginer Dieu le Père sans Fils. Le Fils est

\footnotetext{
${ }^{8}$ Disputatio cum Heracleida 4, 5, trad. J. Scherer, SCh 67, 61-63.

9 De principiis I 2, 2, trad. H. Crouzel et M.M. Simonetti, SCh 252, 113-115.
} 
donc éternel et il est le principe de toute créature et Dieu n'a pas subi un changement: il n'est pas devenu Père, il l'est depuis toujours ${ }^{10}$. Dans ces réflexions sur la génération du Fils, Origène veut éviter tout anthropomorphisme:

„Il n'est pas admissible de comparer Dieu le Père dans la génération de son Fils unique, quand il lui donne l'être, à un homme ou à un animal qui engendre. Mais il faut que cela ait lieu autrement, de manière digne de Dieu, car absolument rien ne peut lui être comparé, non seulement dans la réalité, mais même en pensée, afin que l'homme puisse concevoir comment le Dieu inengendré devient Père du Fils unique. Cette génération éternelle et perpétuelle est comme celle du rayonnement engendrée par la lumière" ${ }^{, 11}$.

Alors, la génération du Fils n'avait rien de matériel, elle est de l'ordre absolument spirituel, elle n'est pas située dans le temps; on ne peut pas s'imaginer Dieu le Père sans Fils, notre auteur compare l'éternité de cette génération au rayonnement de la lumière; quand la lumière luit, il y de rayonnement. Il n'y pas de lumière sans rayonnement.

Origène dans ses cuvres voulait surtout souligner l'existence réelle du Fils:

„Ils s'imaginent savoir clairement ce que signifie le nom de Verbe donné au Fils de Dieu. Surtout, comme ils ne cessent de citer: „Mon cœur a prononcé une bonne Parole» (Ps 44, 2), ils pensent que le Fils de Dieu est une expression du Père qui se trouve, pour ainsi dire, dans les syllabes et, selon ce point de vue, si nous les interrogeons avec précision, ils ne lui accordent ni substance concrète (v́jootáovv) ni existence distincte (oủoż oủoíav) et ne déterminent pas clairement sa nature"12.

Pour Origène il est claire que le Fils n'est pas une expression du Père, non plus sa manifestation; il existe réellement comme un être concret.

Notre théologien aborde le même sujet dans un autre passage de son livre:

„Ce qui trouble beaucoup de personnes qui veulent être pieuses et qui, par crainte

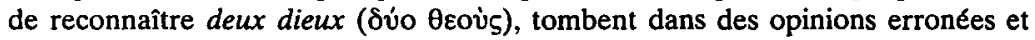
impies, soit que, tout en confessant comme Dieu celui qu'elles appellent Fils au

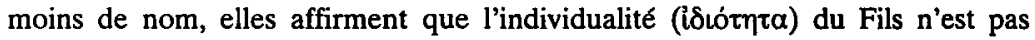
différente de celle du Père, soit que, tout en niant la divinité du Fils, elles admettent que son individualité et la substance personnelle sont, dans leurs caractéristiques propres, différentes de celles du Père"13.

${ }^{10}$ Ibidem I 2, 3, SCh 252, 117: „Celui qui attribue un commencement à la Parole de Dieu et à la Sagesse de Dieu, ne bafoue-t-il pas davantage encore de façon impie le Père inengendré, en lui refusant d'avoir toujours été Père, d'avoir engendré une Parole et eu une Sagesse dans tous les temps et siècles antérieurs, de quelque façon qu'un puisse les nommer?".

11 Ibidem I 2, 4, SCh 252, 119.

12 In Joannem I 151, trad. C. Blanc, SCh 120bis, 135.

13 Ibidem II 16, SCh 120bis, 221. 


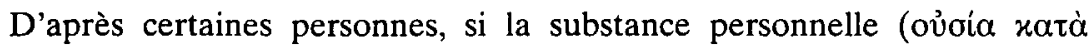

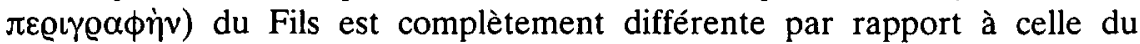
Père, il y a deux Dieux; si cette substance est la même, le Fils n'existe pas réellement, mais il n'est qu'une manifestation du Père. Ces deux opinions sont fausses exhorte Origène:

„Ne pensons pas qu'en appelant le Christ Sagesse de Dieu nous le traitons comme un être sans substance (insubstantiuum), comme si, pour prendre un exemple, nous n'en faisions pas un être animé et sage, mais une sorte de chose qui rendrait sage, en se présentant et en pénétrant dans les intelligences de ceux qui sont devenus capables de recevoir les facultés et la compréhension qu'elle donne. S'il est admis une fois pour toutes que le Fils unique de Dieu est sa Sagesse subsistant de manière substantielle, je ne crois pas que notre pensée pourra désormais s'égarer à soupçonner que son hypostase, c'est-à-dire sa substance ait quelque chose de corporel, puisque tout ce qui est corporel est déterminé par sa forme, sa couleur et sa grandeur" $"$.

Théologien d'Alexandrie soulignait que le Fils est le Verbe du Père (son Logos), mais pas au sens d'une parole prononcée, qui se fait entendre comme un écho et dans ce sens elle existe, mais sans une vraie substance, sans existence distincte. Il n'est pas non plus en tant que Sagesse une qualité de Dieu. Le Fils existe réellement, il a une substance concrète, mais cela ne signifie pas que cette substance soit corporelle ou matérielle, limitée par la forme ou couleur. Cette substance est réelle mais pas matérielle.

Origène comprend que pour certains penseurs il n'est pas facile de s'imaginer comment „une parole prononcée soit un fils"

„Qu'il est étrange de ne pas s'en tenir à la lettre pour tous les autres noms, mais de chercher à comprendre, par exemple, comment Jésus-Christ est une porte, dans quel sens une vigne et pour quel motif un chemin, et de renoncer à faire de même uniquement quand il est appelé Logos"? ${ }^{16}$.

En effet, les noms comme le chemin ou la porte ou la vigne appliqués au Christ ne sont pas utilisés en même sens que le titre de Fils ou de Logos. A travers les Ecritures, Origène montre que les autres titres montrent les différents aspects de la mission du Christ, tandis que le nom „Logos" exprime sa nature, dans ce sens tous les titres appliqués au Christ „sont unis en un seul Logos”17.

Origène croyait profondément que le Fils existait réellement, comme un être distinct du Père, et qu'il est Dieu, mais il était pour lui difficile d'expliquer la relation entre le Père et le Fils. Selon G.L. Prestige, ,la principale source de

\footnotetext{
${ }^{14}$ De principiis I 2, 2, SCh 252, 113.

15 Origène, Commentaire sur St Jean I, 16, SCh 120 bis, 221.

16 Ibidem I154, SCh 120 bis, 137.

17 Ibidem I 196, SCh 120 bis, 157.
} 
difficultés venait du fait que, jusqu'alors, aucune distinction satisfaisante n'avait été établie clairement entre dérivation et création. Aussi longtemps que la déité suprême fut regardée comme un être unitaire, cette lacune n'entraîna pas de conséquences sérieuses, parce que tout objet auquel pouvait être assignée une origine était aussi une créature. Mais quand on vit à considérer la déité comme une triade et à distinguer, au sein de l'être divin lui-même, une seconde et une troisième Personne, le problème de la dérivation, distincte de la création devait surgir [...] Comment la triade peut-elle se concilier avec la monarchie?"18.

Dans les œuvres d'Origène il y a des passages où il présente le Christ comme égal par rapport au Père:

„Le Père et le Fils ont une seule et même toute-puissance, de même que le Seigneur lui-même est un seul et même Dieu avec le Père [...] De même que personne ne doit se choquer, puisque le Père est Dieu, de ce que le Seigneur aussi soit Dieu; de même, puisque le Père est dit tout-puissant, personne de doit se choquer de ce que le Fils de Dieu soit dit tout-puissant. Sera pareillement ce qu'il dit lui-même au Père: «Tout ce qui est à moi est à toi, et tout ce qui est à toi est à moi, et je suis glorifié en eux" $(\mathrm{Jn} 17,10)$. Si vraiment tout ce qui est du Père est du Christ, parmi ce qu'est le Père se trouve aussi la toute-puissance. Sans aucun doute le Fils unique lui-même doit être tout-puissant pour que tout ce qu'a le Père, le Fils l'ait pareillement"19.

D'après cette explication, il n'y a pas de différence entre le Fils et le Père, mais il s'agit surtout d'un aspect: la puissance. Justement, le Fils possède la même puissance que le Père comme il est Dieu comme le Père. Mais dans d'autres passages, la vision du Christ par rapport au Père est moins claire.

Origène se referait toujours à la Bible, même si parfois il lui imposait une interprétation défectueuse. Il a remarqué une différence dans le Nouveau Testament dans la façon de parler de la divinité du Père et du Fils:

„C'est avec une grande attention et non comme un homme ignorant la précision rigoureuse de la langue grecque que Jean utilise l'article dans certains cas et le passe sous silence dans d'autres: devant le Logos, il place «le» et devant le mot "Dieu», tantôt il le met et tantôt il le supprime. Il met l'article lorsque le nom de Dieu désigne l'Inengendré, cause de l'univers, il le laisse de côté lorsque le Logos est appelé Dieu"20.

Il donne une explication de cette façon de se servir de l'article défini «le» (ó):

„Il faut dire d'une part que le Dieu avec article (ó $\theta \varepsilon o ́ \varsigma)$, c'est Dieu même

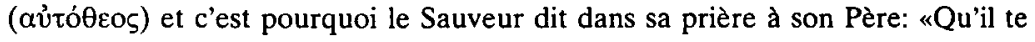

${ }^{18}$ G.L. Prestige, Dieu dans la pensée patristque, Paris Aubier 1955, 126.

19 De principiis I 2, 10, SCh 252, 135.

20 In Joannem II 16, SCh 120bis, 221. 


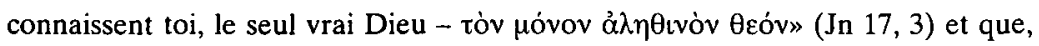
d'autre part, tout ce qui en dehors de Dieu même, est déifié par participation à sa divinité, il serait plus juste de ne pas l'appeler le Dieu (ó $\theta \varepsilon o ́ s)$ mais simplement

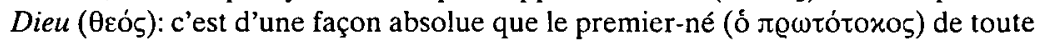
créature, parce qu'il demeure auprès de Dieu et qu'il est pour ce motif le premier à s'imprégner de sa divinité, est plus digne d'honneur que ceux qui, en dehors de lui, sont dieux - et dont Dieu est le Dieu selon la parole: „le Dieu des dieux, le Seigneur a parlé et il a convoqué la terre» (Ps 49, 50) - de devenir des dieux, en puisant auprès de Dieu de quoi les déifier et, dans sa bonté, leur en faisant part avec libéralité" 21 .

Origène suppose que quand Jean dans le Prologue dit que „le Verbe était

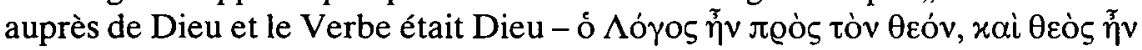
ó $\Lambda$ ó Ş" (Jn 1,1), il veut donner à comprendre que le Logos n'est pas Dieu de la même manière que le Père. Quand il s'agit de Dieu le Père, St Jean met l'article défini; quand il s'agit du Logos, il dit seulement $\mathrm{Dieu}$, sans article; on ne dit pas: Le Verbe était le Dieu, mais le Verbe était Dieu. Cette façon de parler devrait, d'après Origène, signifier une différence entre le Père et le Fils quant à la divinité. Dieu le Père est le vrai Dieu, le Dieu en lui-même ( $\alpha$ vóócos), tandis que le Fils est Dieu par la participation à la divinité du Père

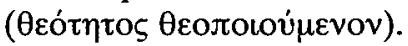

Ce qui est frappant dans cette explication, c'est le mot dieux au pluriel. Ce mot est utilisé d'après le Psaume 49 où justement se trouve une expression "le Dieu des dieux". D'après Origène, il ne s'agit pas des dieux païens (parce que le Dieu d'Isral n'est pas Dieu des dieux païens), mais ce terme signifie ceux qui sont déifiés, ceux qui participent à la vie de Dieu. Eux, dans un sens, sont des dieux par participation, ils n'ont pas la divinité en eux-même, mais ils l'ont acquise.

Ce qui est surtout étonnant, Origène semble mettre à côté des personnes déifiées aussi le Fils: „c'est d'une façon absolue que le premier-né de toute créature, parce qu'il demeure auprès de Dieu et qu'il est pour ce motif le premier à s'imprégner de sa divinité". Est-ce que cela signifie que le Fils est Dieu parce qu'il est imprégné par la divinité du Père? Il est vrai qu'il occupe une place particulière, mais en fait il participe à la divinité du Père comme les autres qui sont déifiés. Sûrement, en tant que le premier-né, il a accès à la divinité du Père en première place et peut-être dans la mesure plus grande que les autres. „Il est plus digne d'honneur qu'eux”, mais il semble que la différence entre lui et les autres n'est pas grande; elle est plutôt quantitative, pas tellement qualitative. Le Fils est appelé le premier-né, mais de toute créature ce qui pourrait être compris comme s'il était une créature lui-même aussi. En lisant cette explication d'Origène, on pourrait avoir l'impression que le Fils se trouve

21 Ibidem II 17, SCh 120bis, 223. 
plutôt à côté des créatures qu'à côté du Père. En fait Origène n'a jamais enseigné que le Fils était une créature, mais certaines de ses constatations se laissent comprendre ainsi ${ }^{22}$.

Chez Origène on trouve d'autres passages qui se laissent interpréter au sens défavorable pour le Fils:
„Dieu est donc le vrai Dieu. Les dieux qui sont formés d'après lui sont comme les reproductions d'un prototype; mais d'autre part, l'image archétype de ces multi- ples images, c'est le Logos qui est auprès de Dieu, qui était dans le principe, qui, parce qu'il est auprès de Dieu, demeure toujours Dieu, car il ne serait pas Dieu s'il n'était auprès de Dieu et il ne demeurerait pas Dieu s'il ne persévérait pas dans la contemplation ininterrompue des profondeurs du Père"23.

Selon Danielou „ce texte nous jette au cœur de la vision d'Origène. Nous voyons d'une part l'opposition établie entre le Dieu avec l'article qui est seul

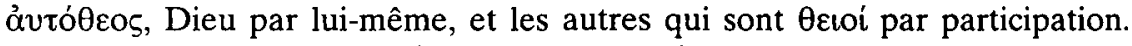
Ceci est inspiré de Philon (De somniis I 230). En ce sens, le Père seul est $\dot{\alpha} \lambda \eta \theta$ vvò $\varsigma$ $\theta \varepsilon \dot{\varsigma} \varsigma$ et par conséquent transcendant au Fils. Origène veut rassurer par là ceux que la crainte du polythéisme fait tomber dans le modalisme ou l'adoptianisme, mais il ne le fait qu'en attribuant au Fils une divinité participée, comme celle de toutes les créatures spirituelles, qui sont des $\theta \varepsilon ı$. Mais, par

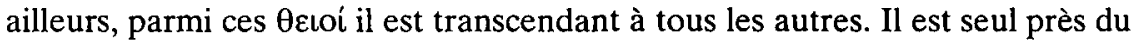
Père, seul il fait toute sa volonté. Il ne possède pas la divinité de lui-même, il la reçoit du Père, mais à son tour, c'est de lui que vient toute divinisation. Il est donc d'un autre ordre que le Père, il est aussi d'un autre ordre que les

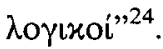

Selon Origène, le Père est le vrai Dieu et tous les autres qui sont appelés

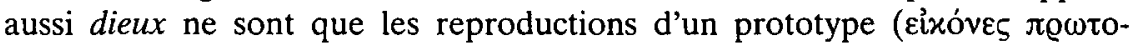

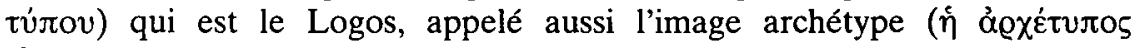

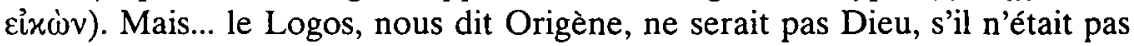
auprès de Dieu et il ne demeurerait pas Dieu, s'il ne persévérait pas dans la contemplation des profondeurs du Père. Il est Dieu parce qu'il se trouve auprès de Dieu et parce qu'il persévère dans la contemplation du Père. Si on continue cette réflexion, on pourrait dire que si le Logos s'arrête de contempler le Père, il cesserait d'être Dieu! Sûrement, Origène ne prenait pas en considération une telle possibilité, mais théoriquement il semble possible pour le Logos de se détourner du Père et de perdre sa dignité divine, car il ne l'a pas en lui-même,

${ }^{22}$ Voici un passage qui se laisse comprendre dans ce sens: „Nous avons donc compris comment la Sagesse est le principe des voies de Dieu et comment elle est dite créée, en tant qu'elle préforme et contient en elle les espèces et les raisons de toute la création" (De principiis I 2, 3, SCh $252,115)$.

23 In Joannem II 18, SCh 120bis, 223.

24 J. Danielou, Origène, Paris 1948, 251. 
mais il l'a possède parce qu'il demeure auprès de Dieu. Une telle vision qui attribue la vraie divinité seulement au Père et diminue la position du Fils est typique pour le courant de pensée appelé subordinatianisme.

Origène se rendait compte que ses explications pourraient être étonnantes:

„Il est vraisemblable que certains seront choqués de ce que nous avons dit, à savoir que le seul Père est proclamé vrai Dieu et que, cependant, à côté du vrai Dieu, plusieurs deviennent dieux par participation à Dieu; ils craindront de ravaler la gloire de celui qui dépasse toute créature au niveau des autres êtres qui reçoivent le titre de dieux"

Pour faire mieux comprendre son point de vue, il explique pourquoi donner aux êtres créés le titre de dieux, ce qui n'était pas conforme à la tradition chrétienne. Il introduit deux notions du logos:

„Le logos qui est en chacun des êtres doués de raison a avec le Logos demeurant dans le principe auprès de Dieu, qui est le Logos de Dieu, les mêmes rapports que le Dieu Logos avec Dieu le Père. Car ce que le Père, Dieu même et vrai Dieu, est à l'égard de son image et des images de son image - on dit en effet que les hommes sont selon l'image et non images, le Logos même l'est à l'égard du logos qui est en chaque créature"26.

Alors il y a le vrai Dieu le Père et son Logos qui est son image ${ }^{27}$. Ensuite chaque être doué de raison a un logos en lui même. La relation du Logos de Dieu à Dieu le Père est comme la relation du logos d'une créature au Logos de Dieu. Comme le Logos de Dieu est l'image de Dieu le Père, ainsi le logos d'une créature est l'image du Logos de Dieu. On voit dans ce raisonnement une vision descendante: Dieu le Père, le vrai Dieu qui est au sommet de tout, puis

25 In Joannem II 19, SCh 120bis, 225.

${ }^{26}$ Ibidem II 20, SCh 120bis, 225.

${ }^{27}$ De principiis I 2, 8, SCh 252, 127-129: „Pour comprendre plus pleinement comment le Sauveur est une figure de la substance ou subsistance de Dieu, prenons un exemple qui, sans exprimer complètement ni proprement ce dont il s'agit, est choisi cependant parce que le Fils qui était sous la forme de Dieu s'est dépouillé de lui-même et, par ce dépouillement, a cherché à nous montrer la plénitude de la divinité. Supposons qu'on ait fait une statue tellement grande qu'elle contiendrait par sa grandeur le monde entier et qu'elle ne pourrait être vue de personne à cause de son immensité, et qu'on ait fait une seconde statue, en tout semblable à l'autre par la configuration des membres et les traits du visage, par la forme et la matière, sans être cependant aussi grande. Ceux qui ne pourraient pas examiner et contempler la première à cause de son immensité pourraient du moins, en voyant la petite, croire qu'ils ont vu la grande, puisqu'elles seraient absolument semblables par le dessin des membres et du visage, par la forme et la matière. Parallèlement le Fils, se dépouillant de son égalité avec le Père pour nous montrer le chemin de la connaissance, devient la figure et expression de sa substance [...]. Le Fils de Dieu, inséré dans la forme minuscule d'un corps humain, indiquait en lui-même, par suite de la similitude de ses œuvres et de sa puissance, la grandeur infinie et invisible de Dieu le Père présent en lui et c'est pourquoi il disait à ses disciples: "Qui m'a vu a vu le Père» (Jn 14, 9)". 
le Logos qui est à son image, et ensuite les êtres créés qui sont les images de l'image principale (= du Logos de Dieu). Dans ce contexte, on peut comprendre la suite de la pensée d'Origène:

„Car l'un et l'autre a le rôle de source: le Père - de la divinité; le Fils du logos (des créatures). De même donc qu'il y a beaucoup de dieux, mais nous n'avons qu'un seul Dieu, le Père, et qu'il y a beaucoup de seigneurs, mais nous n'avons qu'un seul Seigneur, Jésus-Christ, de même il y a beaucoup de logoi, mais nous prions pour posséder le Logos qui était dans le principe auprès de Dieu, le Dieu Logos"28.

Ainsi le Père est l'unique source de la divinité, et le Fils participe à cette source. Le Fils est la source des logoi des créatures; ces logoi participent à la source qui est le Logos principal. Ceux qui possèdent un logos, ont quelque chose de Dieu, ils peuvent - selon Origène - être appelés dieux, on peut appeler un être créé dieu, mais pas dans le même sens que Dieu le Père ni que le Fils. Car „le Dieu de l'univers est le Dieu des élus et, bien plus encore, le Dieu du Sauveur des élus; il est aussi le Dieu de ceux qui sont véritablement des dieux, et d'une manière générale, le Dieu des vivants et non pas des morts" ${ }^{29}$.

Dieu le Père est toujours vu comme le vrai Dieu, comme Dieu du Fils (du Sauveur) et Dieu de ceux qui sont élus et appelés dieux au sens de participer à la divinité, d'abord du Fils et par lui à celle du Père. Le Fils selon Origène, n'est pas Dieu dans le même sens que le Père:

„Cependant, bien qu'il transcende par son essence, sa dignité, puissance, sa divinité - il est, en effet, le Verbe vivant - et sa sagesse, tant d'êtres si admirables, cependant il n'est en rien comparable à son Père. Il est, en effet, l'image de sa bonté et le rayonnement, non de Dieu, mais de sa gloire et de sa lumière éternelle, l'exhalaison, non du Père, mais de sa puissance, la pure émanation de sa gloire de Tout-Puissant, le miroir sans tache de son activité, miroir à travers lequel Paul, Pierre et leurs semblables voient Dieu, car il a dit: «Qui m'a vu, a vu le Père qui m'a envoyé» (Jn 14,9)"30.

Comme nous le voyons, dans toutes ces explications le Christ est placé au milieu entre Dieu le Père et autres êtres. D'après Origène, „bien que le Fils surpasse tous les trônes et dominations et tout nom qui est nommé en ce monde ou dans le monde à venir, en substance, dignité, pouvoir, divinité et sagesse, il ne peut cependant être comparé au Père sur aucun point. Car, par rapport au Père, le Logos n'est qu'une image de sa bonté et de sa splendeur, et non, chose étrange, de Dieu, mais seulement de sa gloire et de sa lumière éternelle; si fidèlement qu'il reflète Dieu le Père, il n'est qu'un miroir. La seule justification que l'on pourrait tenter, d'un tel éclat, du côté orthodoxe serait de soutenir

${ }^{28}$ In Joannem II 20, SCh 120bis, 227.

29 Ibidem II 24, SCh 120bis, 229.

30 Ibidem XIII 152, SCh 222, 115 
qu'Origène a dans l'esprit la monarchie au sens strict. Dans la mesure où le Logos jouit de la nature divine et la révèle, il dispense une richesse qui n'est plus la sienne propre par origine et, à ce point de vue, il est incomparable au Père dont la gloire dérive",31.

2. Dans la doctrine sur l'Esprit-Saint. Le subordinatianisme d'Origène est devenu encore plus visible, quand il parlait de l'Esprit Saint. D'abord, il constate que „de nombreux passages des Ecritures nous apprennent qu'il a un Saint-Esprit" ${ }^{\text {32 }}$. On le voit déjà dans le livre de la Genèse:

„L'Esprit de Dieu qui se déplaçait sur les eaux, comme il est écrit, au début de la création du monde, je ne le crois pas autre que l'Esprit-Saint, selon ce que je puis comprendre" ${ }^{33}$.

Ensuite il souligne que „quant à l'être substantiel qu'est l'Esprit-Saint, personne n'a pu en avoir le moindre soupçon"34, donc l'Esprit Saint existe comme un être qui a sa substance propre (subsistentia Spiritus Sancti), pas comme un esprit de Dieu au sens impersonnel. Mais notre théologien commence à avoir des doutes quant à l'origine de l'Esprit-Saint:

„Jusqu'à présent nous n'avons pu trouver dans les Ecritures saintes aucune parole disant que le Saint Esprit ait été fait ou créé"35.

Une telle constatation signifie qu'Origène voyait l'Esprit-Saint comme un être pas tout à fait comparable au Père et au Fils quant à la dignité, car ni le terme factura ni creatura qu'il utilise [si Rufin les a traduits à la lettre] ne convient pas à Dieu $^{36}$. Donc, l'Esprit-Saint est inférieur par rapport au Père et au Fils. Origène continue ses réflexions dans ce sens:

„Il ne faut pas prendre comme une sorte de blasphème la phrase: «Personne n'est bon si ce n'est pas un seul, Dieu le Père» (Mc 10,18), comme si on y voyait une négation de la bonté du Christ ou de l'Esprit-Saint. Mais, comme on l'a dit plus haut, il faut placer dans le Père la bonté dans son principe; le Fils qui en naît (Filius natus) ou l'Esprit-Saint qui en procède (Spiritus Sanctus procedens) reproduisent en eux, sans aucun doute, la nature de cette bonté qui est dans la source d'où le Fils est né (natus) et d'où l'Esprit-Saint procède (procedit)"37.

${ }^{31}$ G.L. Prestige, op. cité, p. 125.

32 De principiis I 3, 2, SCh 252, 145.

33 Ibidem I 3, 3, SCh 252, 149.

34 Ibidem I 3, 1, SCh 252, 145.

${ }^{35}$ Ibidem I 3,3, SCh 252, 149: „Nullum sermonem in scripturis sanctis invenire potuimus, per quem Spiritus Sanctus factura esse vel creatura diceretur".

${ }^{36}$ A comparer: „En ce qui le (Esprit-Saint) concerne, on ne voit pas clairement s'il est né ou n'est pas né, s’il faut le considérer comme Fils de Dieu ou non" (De principiis Prefatio 4, SCh 252,83 ).

37 Ibidem I 2, 13, SCh 252, 143. 
Alors, Origène utilise [selon la version de Rufin] le mot naître (nasci) par rapport au Fils et du mot procéder (procedere) par rapport au Fils. Ce sont les termes acceptés par la théologie et utilisés jusqu'à nos jours. Mais, même dans cette explication on voit qu'il n'est met pas le Fils et l'Esprit-Saint sur le même niveau; c'est uniquement le Père qui est le principe de la bonté ou la source de la bonté, tandis que le Fils et l'Esprit-Saint participent à cette bonté et la "reproduisent en eux".

Parfois dans la théologie d'Origène on trouve des points inacceptables sur la procession de l'Esprit-Saint:

„S'il est vrai que toutes choses furent par son intermédiaire, il s'agit d'examiner si l'Esprit-Saint fut également par lui. Je pense que, pour quiconque affirme qu'il a une origine et met en avant «toutes choses furent par lui», il sera nécessaire d'admettre que le Saint-Esprit fut, lui aussi, par l'intermédiaire du Logos, car le Logos est plus vénérable que lui" ${ }^{38}$.

Il ne faut pas oublier que ce théologien formulait ses opinions sur l'EspritSaint, ainsi que celles sur le Christ, dans le contexte polémique; avant tout il voulait s'opposer à ceux qui disaient „qu'il n'est pas d'existence propre au Saint-Esprit, différente de celle du Père et du Fils" ${ }^{\text {"39 }}$. Origène admettait l'existence réelle de l'Esprit, mais il le croyait inférieur par rapport au Fils, comme le Fils est inférieur par rapport au Père. Il supposait que l'Esprit fut par

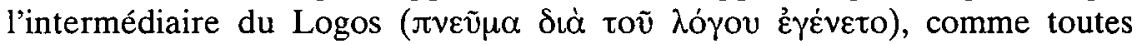
autres choses, et par conséquent il est moins vénérable que le Logos.

Voici une autre explication sur l'Esprit-Saint:

„Pour nous, qui sommes persuadés qu'il y a trois réalités subsistantes distinctes

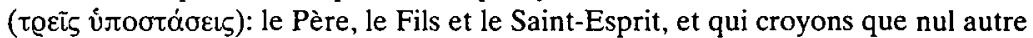
que le Père n'est sans origine, nous tenons pour plus conforme à la piété et pour vrai que, si toutes choses furent par le Logos, le Saint-Esprit a plus de dignité que tout le reste et qu'il est par son rang le premier de tous les êtres suscités par le Père au moyen du Christ. C'est peut-être le motif pour lequel il ne porte pas, lui aussi, le nom de Fils de Dieu, car seul le Fils unique est, dès le commencement, fils par nature et il semble que le Saint-Esprit ait besoin de son intermédiaire pour subsister individuellement, et non seulement pour exister, mais encore pour être sage, intelligent, juste, et tout ce qu'il faut penser qu'il est, puisqu'il participe aux attributs du Christ que nous avons énumérés" ${ }^{40}$.

Ainsi Origène exprime l'opinion que l'Esprit-Saint a une existence réelle

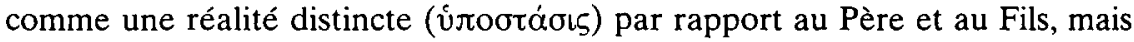
qui non seulement fut par l'intermédiaire du Fils, mais aussi reçoit ses qualité

\footnotetext{
${ }^{38}$ Ibidem II 73, SCh 252, 257.

39 Ibidem II 74, SCh 252, 257.

${ }^{40}$ Ibidem II 74-75, SCh 252, 259.
} 
du Fils; c'est grâce au Fils qu'il est sage, intelligent, juste. L'Esprit a plus de dignité que les autres êtres, mais son rang est moins élevé que celui du Père et du Fils ${ }^{41}$.

Comme nous le voyons, même si Origène parle de la Sainte Trinité ${ }^{42}$, même s'il se réfère au symbole baptismal où la foi au Père, au Fils et au Saint-Esprit était confessée ${ }^{43}$, il garde toujours une vision descendante: le Père en première place, en deuxième le Fils et en troisième l'Esprit-Saint ${ }^{44}$. On comprend mieux la vision d'Origène quand on voit comment il présente la relation entre Dieu et les créatures:

„Nous disons que le Sauveur et le Saint-Esprit transcendent toutes les créatures, non par comparaison, mais par une transcendance absolue, mais qu'il est lui-même transcendé par le Père autant et même davantage que lui-même et le Saint-Esprit transcendent les autres êtres, qui ne sont pas négligeables"

Alors, comme le Fils et l'Esprit-Saint transcendent les créatures, ainsi eux ils sont transcendus par le Père, autrement dit: comme le Fils et l'Esprit sont supérieurs par rapport aux créatures, ainsi le Père est supérieur par rapport à eux ${ }^{46}$.

${ }^{41}$ Parfois Origène présente l'Esprit-Saint comme égal, à certains aspects, au Fils, par exemple: „Il faut penser que comme le Fils, qui seul connaît le Père, le révèle à qui il veut, le Saint-Esprit, qui est seul à scruter jusqu'aux profondeurs de Dieu, révèle Dieu à qui il veut. «Car l'Esprit souffle où il veut» (Jn 3,8). Il ne faut pas penser que l'Esprit connaît lorsque le Fils lui révèle. En effet, si l'Esprit-Saint connaît le Père lorsque le Fils le lui révèle, il passe donc de l'ignorance à la connaissance. Cela est en vérité impie et sot, de le reconnaître Esprit-Saint et de lui attribuer l'ignorance. Il ne faut pas penser qu'il était auparavant autre chose que Saint-Esprit et qu'il a progressé jusqu'à devenir Saint-Esprit. C'est comme si l'on osait dire qu'alors, quand il n'était pas Saint-Esprit, il ignorait le Père et qu'après qu'il en eut reçu la connaissance il est devenu Saint-Esprit. Si cela était, jamais ce Saint-Esprit n'aurait été compris dans l'unité de la Trinité (in unitatem Trinitatis) avec Dieu le Père immuable et avec son Fils; c'est donc qu'il a toujours été Saint-Esprit" (De principiis I 3, 4, SCh 252,151-153). Ainsi Origène constate l'égalité de l'Esprit-Saint par rapport au Fils quant à la connaissance du Père. Il souligne également que l'Esprit-Saint possède sa connaissance du Père depuis toujours, il exclue tout changement dans sa nature.

42 Cfr. De principiis I 3, 5, SCh 252, 153: „Il semble bon de rechercher pourquoi ce qui reçoit par Dieu la régénération pour son salut a besoin du Père, du Fils et du Saint-Esprit, alors qu'il ne recevrait pas le salut sans la Trinité entière (nisi sit integra Trainitas)".

43 Ibidem I 3, 2, SCh 252, 147: „Tout cela nous révèle la grande autorité et dignité qu'a l'Esprit-Saint en tant qu'être substantiel (substantiam Spiritus Sancti), telle que le baptême de salut (salutare baptismum) ne peut être accompli que par l'autorité de la Trinité (Trinitatis auctoritate) la plus excellente de toutes, par l'invocation du Père, du Fils et de l'Esprit-Saint".

44 Cette vision est surtout visible dans la Préface qu'Origène à précédée son Traite des principes (4).

45 In Joannem XIII 151, SCh 222, 115.

46 Il ne faut pas oublier que cette opinion a été exprimée par opposition aux gnostiques qui enseignaient qu'ils sont consubstantiels avec Dieu: „Examinons s'il n'est pas de la pire impiété de

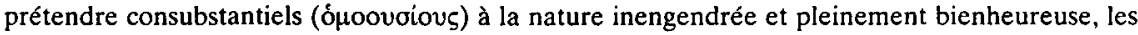


Les théologiens contemporains sont d'accord que „,'est là une remarque très significative, fortement monarchique, en tant qu'elle place en le Père seul les mystères de l'être divin. Elle est fortement subordinatienne, tout au moins en ce sens qu'elle dispose tous les êtres, créés ou divins, selon une hiérarchie d'existence, s'élevant à partir de l'humanité raisonnable, par la création spirituelle des anges et autres êtres célestes semblables, jusqu'au Saint-Esprit et au Christ, et ainsi jusqu'à Dieu le Père. La ligne qui sépare les geneta de Dieu est nettement tirée à partir d'un point qui laisse le Christ et le Saint-Esprit du même côté que les puissances créées et les êtres humains [...]. Au regard superficiel, il peut sembler qu'Origène voulait dire implicitement que le Fils et l'Esprit sont des créatures"47. Pour J. Quasten „il est évident qu'Origène présuppose un ordre hiérarchique dans la Trinité et place le Saint-Esprit à un rang encore inférieur à celui du Fils"48.

\section{VISION DESCENDANTE DANS LA THEOLOGIE EN OCCIDENT}

Pour mieux comprendre la position d'Origène, il faut le situer parmi d'autres théologiens de l'époque, tant en Orient qu'en Occident. L'auteur latin qui a surtout développé la doctrine de Dieu est Tertullien. Chez lui on voit les mêmes tendances. Dans son traité Adversus Praxean, il enseignait:

„Selon cette règle (de foi), je confesse le Père, le Fils et l'Saint-Esprit qui ne sont pas séparés. Un autre est le Père, un autre est le Fils et un autre est l'Esprit-Saint [...]. Le Père est la totale substance (Pater tota substantia est), le Fils est une portion ou dérivée de cette substance (portio et derivatio), comme il a dit lui-même: «Le Père est plus grand que moi» (Jn 14, 28) Alors, le Père est différent du Fils et plus grand que lui (filio maior); un autre qui donne la vie, un autre qui est né; un autre qui envoie et un autre qui est envoyé; un autre qui agit et un autre celui par qui quelque chose est fait" ${ }^{\text {"49. }}$.

Tertullien bien distingue le Père et le Fils et l'Esprit-Saint pour souligner qu'ils existent réellement comme les êtres différents, mais il présente le Père comme substance totale ou principale dont le Fils n'est qu'une dérivée. Il comprend

hommes qui adorent Dieu en esprit et dont Héracleon vient d'affirmer qu'ils sont tombés, puisqu'il dit que la Samaritaine s'est prostituée, bien qu'elle fût de nature spirituelle. Mais ceux qui disent cela ne voient pas que tout ce qui est consubstantiel est susceptible des mêmes attributs; or, si la nature spirituelle, bien que consubstantielle à Dieu, était capable de se prostituer, quelles conséquences sacrilèges, impies, blasphématoire, découlent de leur enseignement sur Dieu, il n'est pas sans danger même de s'imaginer" (In Joannem XIII 148-150, SCh 222, 111-113). Origène voulait montrer qu'une telle opinion était inacceptable, c'est pourquoi il avait souligné que le Père est transcendant (supérieur) même par rapport au Fils et à l'Esprit-Saint.

47 G.L. Prestige, op. cité, p. 127.

48 J. Quasten, Initation aux Pères de l'Eglise, trad. J. Laporte, II, Paris 1956, 98.

49 Adversus Praxean 9, 1-2, CCL 2, 1168, trad. propre du texte latin. 
littéralement ce qui Jésus a dit lui-même: „Le Père est plus grand que moi”. Quant à l'Esprit-Saint, il lui attribue le troisième degré, après le Fils, juste comme Origène ${ }^{50}$.

Afin de mieux présenter les relations entre Dieu le Père et le Fils et l'Esprit-Saint, Tertullien se servait des analogies:

„Dieu a proféré le verbe, ainsi que le proclame le Paraclet lui-même, comme une racine produit des rejetons, comme une sołirce donne naissance à une rivière, et le soleil émet son rayonnement. Ces manifestations sont aussi des émanations des substances dont elles procèdent. Par conséquent, je n'hésite pas à appeler rejeton le fils de la racine, et la rivière la fille de la source, et le rayon le fils du soleil. Toute source, en effet, est un père, et ce qui coule de la source est un engendré. Il en va ainsi particulièrement pour le Verbe de Dieu, lui qui a reçu comme désignation toute spéciale le nom de Fils. Et pourtant, le rejeton n'est pas séparé de la racine, ni la rivière de la source, ni le rayon du soleil. Le Verbe n'est pas davantage séparé. Suivant cette donc la forme de ces analogies, je confesse que j'appelle Dieu et son Verbe - le Père et le Fils - deux. En effet, la racine et le rejeton sont deux choses distinctes, mais réunies. La source et la rivière sont deux manifestations, mais indivisées. De même le soleil et le rayon sont deux objets pour la vue, mais résidant l'un dans l'autre. Tout ce qui procède de quelque chose doit nécessairement être second par rapport à ce dont il procède, sans en être forcément séparé. Là par conséquent où on compte un second, il doit y avoir deux, et là où l'on compte un troisième, il doit y avoir trois. L'Esprit est donc le troisième en partant de Dieu et du Fils, comme le fruit du rejeton est troisième en partant de la racine, ou comme le canal issu de la rivière est troisième en partant de la source, ou enfin comme l'extrémité du rayon est troisième en partant du soleil. Rien cependant n'est étranger à cette source première dont il reçoit ce qui lui appartient. De même la Trinité, en procédant du Père par degrés combinés et conjoints, ne trouble aucunement la monarchie, tandis qu'elle sauvegarde l'état de l'économie"51.

Le théologien souligne la réelle existence du Père et du Fils et de l'Esprit-Saint, il proclame leur unité, mais il place le Père un première place et le Fils en seconde, car - d'après lui - tout ce qui procède doit nécessairement se trouver en seconde place par rapport à celui dont il procède. Comme le Fils procède du Père, il est inférieur par rapport du Père, se trouve en deuxième position. L'Esprit-Saint est inférieur par rapport au Fils, il est toujours placé au troisième degré.

Tertullien, comme Origène et d'autres théologiens, est marqué par une vision monarchique et subordinatienne de Dieu, ce qui était - comme nous avons dit plus haut - typique pour cette époque.

${ }^{50}$ Cfr. ibidem: „Necessitate autem hoc dico cum eundem Patrem et Filium et Spiritum condendunt, adversus oikonomiam monarchiae adulantes [...]. Sic alium a se Paraclitum, quomodo et nos secundum in Filio ut tertium gradum ostenderet in Paraclito".

51 Adversus Praxean 8, 5-7, CCL 2, 1167-1168, trad. J. Quasten, op. cité, t. II, p. 388. 
Terminons nos considérations par quelques observations du prof. Wolinski sur ce sujet: „Tout d'abord la visée de foi des auteurs en question, de Justin à Origène, est une chose et d'adéquation de leurs élaboration conceptuelles à leur sujet en est une autre. Leur foi en la divinité du Fils et de l'Esprit ne saurait être sérieusement mise en cause [...]. Il est néanmoins incontestable que dans leur usage des catégories ils ont cherché comme à tâtons à propos d'un problème particulièrement difficile, sans dominer encore la conceptualité qu'ils mettaient en œuvre. Il faut distinguer deux formes de subordinatianisme.

L'un peut et doit être considéré comme orthodoxe (même s'il n'est pas appelé couramment ainsi). C'est celui qui affirme la priorité du Père, en tant qu'il est la source et la cause de la divinité du Fils et de l'Esprit. En ce sens le Père est "plus grand",ce qu'indique l'ordre révélé des personnes divines, qui pose un premier, un deuxième et un troisième. Ce "subordinatianisme" est compatible avec la confession que le Fils et l'Esprit sont Dieu comme le Père. Ce sera encore celui de Basile de Césarée dans la seconde moitié du IV siècle.

L'autre subordinatianisme tient que le Fils et l'Esprit sont en définitive des créatures du Père, quelle que soit par ailleurs leur suréminence par rapport aux autres créatures. Au plan historique tel est celui qui déclenchera la crise arienne [...].

Le subordinatianisme a plusieurs racines doctrinales. La première est l'approche «économique» du Fils, la seule que connaissaient les Pères anté-nicéens. Le discours sur le Fils ne porte pas, comme après Nicée, sur les relations intra-trinitaires, mais sur les rapports entre d'une part le Père, et d'autre part le Fils en relation au monde. C'est à l'occasion de l'apparition de ce monde que ce Fils devient «Fils», dans le «moment» qui précède logiquement la création, puis dans l'incarnation. La relation Père-Fils est posée en termes subordinatiens parce qu'elle vise le Fils comme Verbe "exposé à l'extérieur», devenu chair et accomplissant la mission reçue du Père. Elle vise le Fils associé à toute l'économie du salut [...]. La réflexion scrutera le rapport d'origine qui rattache le Fils au Père. Le Fils est subordonné au Père, parce qu'il provient de lui. La subordination est le corollaire de la provenance d'un autre, qui, de ce fait, est déclaré «plus ancien».

L'environnement de la philosophie grecque a également contribué au climat «subordinatien» de la première spéculation trinitaire. Ici sont en cause le platonisme et le moyen platonisme, que leur influence ait joué directement à partir de la culture dans laquelle baignaient les Pères, ou qu'elle se soit exercé par l'intermédiaire de Philon, très marqué par cette philosophie. La Triade platonicienne affirme l'inégalité des trois hypostases. La philosophie tient que l'image est inférieur au modèle et admet le jeu des intermédiaires entre l'Un et 
le multiple. Origène, conscient de l'originalité chrétienne du médiateur, fait encore appel au schème du Verbe «intermédiaire» et de l'âme du Christ „intermédiaire> entre le Verbe et la chair. Il adoptera d'autant plus volontiers certaines données de cette culture platonisante qu'il y verra le moyen de s'opposer au modalisme sans nier la divinité du Verbe. Il est plutôt remarquable qu'il ait au rendre compte authentiquement de la divinité et de la génération éternelle du Fils dans ce cadre conceptuel, avant le discernement radical qui s'exercera au IV ${ }^{\mathrm{e}}$ siècle. Car on de doit jamais juger ses affirmations à l'aune du jeu des catégories qui se mettront en place au cours des débats relatifs à Nicée" 52 .

Le subordinatianisme de l'avant Nicée était profondément implanté dans l'époque, cela n'était pas - comme nous voulions montrer - une hérésie, mais plutôt une tendance, une manière de voir: les théologiens imprégnés d'un côté par la philosophie (et des courants d'esprit comme la gnose par exemple) et d'autre côté par le monothéisme de l'Ancien Testament, avaient une vision descendante, c'est pourquoi ils plaçaient tout naturellement les Personnes divines dans un ordre hiérarchique. Leur vision subordinatienne, délicate et modérée, a été amplifiée et presque caricaturée par Arius et ses adhérents.

52 B. Sesboüé - J. Wolinski, Le Dieu du salut. Histoire des dogmes, I, Paris 1994, 229-232. 